Béla Dezsö: Die Histiologie u. Sprossenentwickelung der Tethyen etc. 651

Fig. 29. (Gundlach I/V.) Das Entoderm wird zweireihig, die Sprosse selbst mehr bauchig. Ec En.

Fig. 30. (Gundlach I/V.) Ec En. Das Entoderm wird dreireihig und die Sprosse noch mehr bauchig, bis

Fig. 31. (Gundlach I/V.) die Sprosse rundlich geworden ist.

Fig. 32. (Gundlach I/V.) Das Ectoderm scheidet sich in zwei Zellenschichten. f $\mathrm{a}=$ Faserschichtanlage. klst Sch. = kleinsternige Anlage.

Fig. 33. Sprosse (Gundlach I/V), worin schon die kleinen Sterne in einer Schicht ausentwickelt sind, und hat sich auch das beständige Epithel abgesondert. Mes $=$ Faserschicht. En $=$ Endothel. klst $\mathrm{Sch}=$ kleinsternige Schicht. $\mathrm{Ep}=$ Epithel. $\mathrm{Nb}=$ Nadelbündel.

\title{
Die Muskulatur der menschlichen Mundspalte.
}

Von

\author{
Prof. Dr. Chr. Aeby
}

in Bern.

(Hierzn Tafel XXXIV.)

Ueber den Bau des fleischigen Walles, der die Mundspalte umrandet, sind beinahe ebensoviele Ansichten zu verzeichnen, als Forscher, die ihn zum Gegenstande ihrer Untersuchung gemacht haben. Und deren sind nicht wenige. Sofern es sich dabei nur um die Sonderung peripherischer Faserzüge und um die äussere Abgrenzung sogenannter Muskeleinheiten handelt, ist die Frage ohne tiefere Bedeutung und ihre Lösung zum guten Theile nicht viel mehr als Geschmackssache. Anders gestaltet sie sich, wenn entschieden werden soll, wo die verschiedenen Faserbündel Ursprung und Ende finden, und ob die Mundspalte neben denjenigen, die von aussen her an sie herantreten, auch eigene besitzt. Hier 
handelt es sich ja um die unerlässlichen anatomischen Grundlagen fiir die richtige Betrtheilung der gesammten physiologischen Leistnngsfähigkeit. Man hat das Seelenleben, das sich bei Mensch und Thier an diesem Muskelwalle abspielt, so vielfach und eingehend verfolgt, dass es wohl endlich an der Zeit erscheint, die anatomischen Vorstellungen, von denen man sich dabei leiten liess, einer etwas schärferen Probe auf ihre Zulässigkeit als die bisherige zu unterziehen.

Wer einmal dieses Fasergewirr zu lösen versucht hat, der weiss, dass ron einem gewissen Punkte an die feinste Scalpellspitze und die geschickteste Technik daran zu Schanden werden. Ihm auf diese Weise beizukommen, ist haare Unmöglichkeit. Es giebt nur ein Mittel, das weiter zu führen vermag; die mikroskopische Untersuchnng systematisch angelegter, geschlossener Schnittreilen. Man scheint davon bis jetzt noch keinen Gebrauch gemacht zu haben. Nachdem ich es getlian, hoffe ich, nicht allein fiir den menschlichen Organismus an die Stelle der bisherigen eine bessere Erkenntniss setzen zu können, sondern namentlich auch zu vergleichend anatomischer Untersuchung anzuregen. Ist es schon an und für sich von Interesse, die Differenzirungsgeschichte einer dem Seelenleben so nahe stehenden Muskelgruppe zu verfolgen, so ist es hinwiederum nicht unberechtigt, von möglicherweise einfacheren und durchsichtigeren Formen bei Thieren eine Förderung des Verständnisses für die so verwickelten des Menschen zu erwarten.

Bei Muskeln ist eine Aenderung der Architectur während des Wachsthums kaum anzunehmen. Meine Untersuchungen beschränkten sich daher aus technischen Gründen auf die Mundtheile von Neugeborenen. Diese werden in Alkohol gehärtet und dann vermittelst des Mikrotomes in passende Scheiben zerlegt. Senkrecht und parallel zur Mundspalte gefiihrte Dickenschnitte erweisen sich am dankbarsten. Mit Flächenschnitten ist wenig anzufangen und Schrägschnitte lohnen nur ausnahmsweise die Arbeit.

Ueber die systematische Eintheilung der Mundmuskulatur kann ich mich kurz fassen. Wie sich auch deren feineres Geftige verhalten mag, aus praktischen Gründen wird es immer zweckmässig sein, den fleischigen Randwulst der Mundspalte zu einem Ringmuskel (M. orbicularis) zu gestalten und die ausserhalb gelagerten selbständigen Faserzige als ebensoviele Muskeln besonders 
zu benennen. Von solchen anerkenne ich, um mich der einfachsten Nomenklatur zu bedienen, die nach den Lippenrändern auslaufenden Quadrati, sowie die strahlig zum Mundwinkel gestellten Triangulares sup. und inf., den Zygomaticus und Risorius. Alles, was sonst noch an Muskeln beschrieben und benannt worden ist, geht ohne Zwang in den genannten auf.

Der Knotenpunkt der ganzen Angelegenheit liegt am Mundwinkel. Hier treffen die sämmtlichen Muskeln der seitlichen Gesichtsgegend mit dem Ringmuskel zusammen. Wie stehen sie zu einander? Halten sich ihre Fasern von einander gesondert oder laufen sie ohne Unterbrechung in einander fort? Jede der beiden Auffassungen hat ihre Anhänger gefunden. Ich selbst ${ }^{1}$ ) habe mich

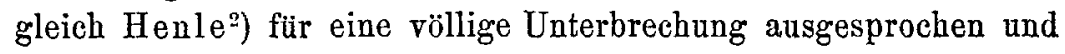
die gegenseitige Verknuipfung einem eingeschobenen Sehnenknoten zugeschrieben. $\mathrm{Henke}^{3}$ ) nimmt zwar im allgemeinen den entgegesetzten Standpunkt ein, bekennt sich aber docb wenigstens zur Annahme einiger sehniger Commissuren. Nachdem ich jetzt das betreffende Gebiet nach allen Richtungen auf das sorgfältigste mikroskopisch durchforscht habe, kann ich nicht einmal solchen beschränkten Commissuren, geschweige denn einem umfänglicheren Sehnenknoten die geringste Berechtigung zugestehen. Zygomaticus und Risorius entbehren iiberhaupt jeglicher Fortsetzung über den Mundwinkel hinaus. Sie enden, wie später des genauern dargethan werden soll, an ihm selbst. Anders die beiden Triangulares und der Buccinatorius. Diese dringen ohne irgendwelche Unterbrechung nach vorgängiger Durchkreuzung ihrer Faserzüge vor, der Triangularis inf. und die ihm benachbarte Hälfte des Buccinatorius in die Oberlippe, der Triangularis sup. sammt dem Reste des Buccinatorius in die Unterlippe ${ }^{4}$ ). Sie allein bilden somit die Grundlage für den Orbicularis. Zygomaticus und Risorius haben an diesem

1) Der Bau des menschlichen Körpers. Leipzig, 1871.

2) Handbuch der systematischen Anatomie des Menschen. Bd. 1. Braunschweig, 1855.

3) Die obern und untern Muskeln der Lippen. Zeitschrift für Anatomie und Entwicklungschichte. 1. Jahrg. Leipzig, 1875.

4) Ich lasse es dahingestellt, ob und in welcher Ausdehnung Randfaserbündel des Buccinatorius der Kreuzung entgehen. Für die Architectur des Orbicularis ist die Frage von untergeordnetem Belange. 
keinen Theil. Ebensowenig ist ein besonderes, an den Mandwinkeln geschlossenes Ringfasersystem vorhanden. Henke glaubte freilich schwache Andeatungen eines solchen gesehen zu haben und ich selbst huldigte lange Zeit derselben Meinung, bevor ich mich entschliessen konnte, sie als irrthïmlich fallen zu lassen. Die dem Lippenrande parallel verlaufenden, und, namentlich in Querschnitten, überall äusserst scharf hervortretenden Muskelbündel gehen an Durchschnitten in der Gegend des Mundwinkels stets eine Strecke weit verloren, was mit der Vorstellung eines geschlossenen Ringmuskels schlechterdings unverträglich ist. Der vielfach behauptete Faseraustausch am Aussenrande von Zygomaticus und Triangularis inf., sowie zwischen diesem und dem Triangularis sup. findet ganz bestimmt nicht statt und Henke hat in dieser Beziehung vollkommen richtig gesehen. Die Triangulares tiberantworten ihre sämmtlichen Fasern dem Orbicularis.

Es geschieht dies folgendermaassen. Die im ganzen zu platt cylindrischer Masse geordneten Bündel der beiden Triangulares durchflechten sich am Mundwinkel in ziemlich inniger Weise und erzeugen dadurch jenen derben und prallen Knoten, der zwischen Haut und Schleimhaut beim Lebenden wie Todten so deutlich durchzufühlen ist. Zunïchst der letztern treten auch die Buccinatoriusfasern in ihn ein und erhöhen das Gewirre der nach verschiedenen Richtungen sich durchkreuzenden Fasergruppen. Diese durchflechten sich namentlich in den tiefern Parthien mattenartig; wenn gleich mit geringer Regelmässigkeit, und ordnen sich erst beim Uebertritte auf die Lippen wieder zu parallelen Strängen. An der Unterlippe geschieht dies in vollständigerer Weise als an der Oberlippe, wo die Neigung zu spitzwinkliger Durohflechtung fordauert. Jene bringt daher auf Durchschnitten die einzelnen Bündel im allgemeinen schärfer und selbständiger zur Geltung als diese. Die Herkunft derselben lässt sich keineswegs uberall mit voller Sicherheit nachweisen, doch ist deutlich zu erkennen, wie es ausschliesslich Buccinatoriusfasern sind, welehe als kräftiges Bündel dem Lippenrande zunächst zu liegen kommen. Sie rollen sich am Mundwinkel in steilem Bogen, ja oft in der Wendung eines Viertelkreises um die vereinigten Massen der Triangulares herum nach vorn. Sie gestalten sich zu einer nach der Haut hin vorspringenden Randleiste des Orbicularis und reichen bis dicht an die erste Reihe der Haarbälge heran, doch obne sie irgendwo zu 
tiberschreiten (Fig. 1-3). Sie bilden somit die Unterlage des rothen Lippensaumes als der in der Regel völlig drüsen- und haarlosen Uebergangszone von der Haut zur Schleimhaut.

Der Ringmuskel des Mundes entbehrt also der Selbständigkeit. Er ist die unmittelbare Fortsetzung des Buccinatorius and der beiden Triangulares. Somit befinden wir uns wenigtens mit einem Theile der bisherigen Forscher in Uebereinstimmung. Es handelte sich nur darum, die neu gewonnene Erfahrung zu Gunsten einer der bereits bestehenden Añsichten anderen gegentiber in die Waagschale zu werfen. Weiterhin bahnen wir eigene Pfade; denn alles, was bisher über das Verhalten des Orbicularis im Bereiche der Lippen ist veröffentlicht worden, streift wohl die Wahrheit in einzelnen Punkten, bringt sie aber keineswegs voll zur Geltung. Anders als mit Hülfe des Mikroskopes war solches auch gar nicht zu erreichen.

Zwei Ansichten stehen sich schroff gegenïber. Nach der einen, und diese zählt die Mehrzahl der Sehriftsteller zu ihren Anhängern, fliessen die Fasern des Orbicularis von beiden Seiten her in der Mittellinie continuirlich zusammen, nach der anderen, welche Langer ) als Urheber nennt, findet dies nicht statt und eine Endigung in der Haut wird dann für alle zur Nothwendigkeit. Manche von ihnen sollen dabei auf die gegentiberliegende Seite treten und sich mit den von dieser ausgehenden in der Mittellinie aberkreuzen. Beide Ansichten treffen das Richtige nur zur Hälfte. Der Orbicularis enthält eben durchaus ungleichartige Elemente. Die einen sind wirklich durchgehende und bleiben ihm in der ganzen Länge der Lippe getreu, die anderen gehören ihm nur streckenweise an und treten später zur Haut uber. Ersteres gilt vor allem für die Faserztige des Lippenrandes. Von diesen tritt kein einziger mit der Haut in nähere Beziehung und sie überschreiten die Mittellinie ohne alle Unterbrechung. In etwas grösserer Entfernung von der Mundspalte folgen nur noch die tiefern Faserlagen diesem Beispiele, die oberflächlicheren gehen zur Haut (Fig. 4). Schliesslich thun auch jene das Gleiche, so dass in den äusseren Parthien des Muskels durchgehende Fasern liberhaupt gar nicht mehr vorkommen (Fig. 5).

1) Ueber den Musculus orbicularis oris. Medicin. Jahrbücher, Zeitschrift der k. k. Gesellschaft der Aerzte in Wien, 1861. - Ferner: Lebrbuch der Anatomie. Wien 1865. 
Der Charakter der Orbicularisfasern schlägt somit von innen nach aussen ins Gegentheil um. Worauf beruht dies? Wir haben uns allerdings zu dem Geständnisse genöthigt gesehen, dass es nicht möglich sei, die Herkunft der Orbicularisfasern durchweg festzustellen. Aber bedenken wir, dass diejenigen des freien oder inneren Randes unstreitig dem Buccinatorius angehören und dass dieser letztere in seiner ganzen Anordnung eine tiefere Lage besitzt als die beiden Triangulares, so muss es zum Mindesten als höchst wahrscheinlich gelten, dass alle durchgèhenden Fasern Angehörige des ersteren, alle zur Haut gehenden solche der letzteren seien. Beide Gruppen würden dann zur Bildung der mittleren Abschnitte des Orbicularis gemeinschaftlich beitragen. Den Innenrand dagegen zunächst der Mundspalte besässen ausschliesslich Angehörige des Buccinatorius, den entgegengesetzten Aussenrand solche der Triangulares.

Von Wichtigkeit ist die Thatsache, dass der Uebertritt der Triangularisfasern in die Haut nicht in der ganzen Breite der Lippen erfolgt, sondern sich im allgemeinen auf deren Mitte beschränkt. Dabei gilt auch als Regel, dass die Fasern nicht auf der Seite ihres Ursprungs, sondern auf der entgegengesetzten, also nicht diesseits, sondern jenseits der Medianebene ihr Ende finden. In letzterer liberkreuzen sich die beidseitigen Gruppen in ungemein zierlicher und regelmässiger Weise (Fig. 4 und 5) und zwar so, dass ihre Fasern mit zunehmender Entfernung vom freien Lippenrande auch weiter über die Mittellinie hinausgreifen. Ibre ersten Ansätze kommen immer dicht neben die letztere zu liegen. Während somit gekreuzte Fasern am freien Lippensaume gänzlich fehlen, erweitert sich ihr Gebiet nach dem angewachsenen Lippenrande hin in sagittaler und transversaler Richtung mehr und mehr. Im allgemeinen entspricht es dem von den beidseitigen Quadrati eingefassten Raum, doch sei gleich bemerkt, dass es dessen Grenzen eine Strecke weit überschreitet und dadurch mit den Randparthien der genannten Muskeln zusammenfällt. An der Unterlippe ist die Kreuzung weitaus zierlicher und klarer als an der Oberlippe. Die Bündel liegen eben dort weniger dicht beisammen als hier. Zudem sind sie regelmässiger angeordnet und kräftiger gehalten. Longitudinale Dickenschnitte durch die Unterlippe in verschiedener Entfernung von dem freien Rande empfehlen sich daher ganz besonders zur Darlegung diesser merkwürdigen Verhältnisse. 
Einem etwas gelibteren Auge bietet auch die Oberlippe volle $\mathrm{Be}$ friedigung.

Nicht alle Fasern der Triangulares gelangen zur Kreuzung. Eine Minderzahl endet auf der Seite des eigenen Ursprungs, doch immerhin dicht an der Medianebene. Die Thatsache ist an der Unterlippe leicht festzustellen. Umgekehrt giebt es auch Fasern, die nach der Kreuzung bis in die Nähe des gegenüberliegenden Mundwinkels vordringen. Thre Zahl ist immer nur eine geringe und kömmt neben derjenigen der Fasern, die sich in der Nähe der Medianebene halten, kaum in Betracht.

Die Ausstrahlung der zuvor einander parallelen Fasern gesehieht in Fächerform. Die ersten laufen in ziemlich steilem Bogen fast senkrecht zur Haut nach vorn, die folgenden beschreiben immer flachere Bogen und treffen unter immer spitzerem Winkel auf ihr Ziel.

Ganz anders als die Triangulares verhält sich der Zygomaticus. Derselbe betheiligt sich gar nicht, wie man allgemein anzunehmen scheint, an der Bildung des Orbicularis. Er endet vielmehr am Mundwinkel selbst, und zwar gleichzeitig an dessen Aussen- und Innenfläche. Er zerfällt am Aussenrande der sich kreuzenden Triangulares in ein oberflächliches, schwächeres und ein tieferes, stärkeres Bündel (Fig. 6). Jenes geht vor den Triangulares vorbei direct zur Haut. Dieses dringt durch die Triangulares hindurch zur Tiefe und legt sich so dicht an den Buccinatorius an, dass es mit ihm eine einheitliche Masse zu bilden scheint. Nichtsdestoweniger ist seinen Fasern ein anderes Ziel als denen seines Genossen gesetzt. Die einen durchbrechen, wie an gelungenen Horizontalschnitten mit grosser Deutlichkeit zu sehen ist, spitzwinklig die Buccinatoriusschicht, um in der Schleimhant der Umgebung des Mundwinkels zu enden. Die anderen umschlingen das Triangularisbündel in steilem Bogen von innen her nach vorn und treten zur Haut. In Gemeinschaft mit dem vorderen Bündel des Zygomaticus bilden sie somit eine Schlinge, durch welche die vereinigten Massen der beiden Triangulares hindurchlaufen. Da, wie bereits angegeben wurde, die Fasern des Buccinatorius am Mundwinkel ebenfalls in starkem Bogen nach vorn ziehen, so liegt, zumal auf Horizontalschnitten, die Gefahr nahe, sie mit Angehörigen des Zygomaticus zu verwechseln. Die verschiedene Endigungsweise unterscheidet sie bestimmt. Der Zygomaticus rollt den Mund- 
winkel nach hinten und aussen und zieht die ihm zunäichst liegende Schleimhautfäche trichterförmig ein. Sein Werk ist es, wenn manche Schriftsteller einen Theil der Buccinatoriusfasern in die Schleimhaut des Mundwinkels ausstrahlen lassen. Solches geschieht, wie ich an zahlreichen Schnitten zu beobachten Gelegenheit hatte, niemals.

Wenig ist über den Risorius zu sagen. Soviel ich gesehen habe, enden seine Fasern, nachdem sie die Vorderflïche der Triangulares uberkreuzt haben, seitlich in der Haut des Mundwinkels.

Risorius und Zygomaticus gehören also zu einem ganz andern Fasersystem als die Triangulares und es ist durchans fehlerhaft, sie den letztern desshalb beizugesellen, weil sie mit ibnen am Mundwinkel in radiïrer Anordnung zusammentreffen. Durch ihre Endigungsweise bekennen sie sich unzweideutig als Verwandte der Quadrati und gleichsam als Bindeglieder zwischen Quadratus sup. und inf.

Henke behauptet von den letztern, dass sie rom Orbicularis durchaus unabhängig seien und ihre sämmtlichen Fasern vor demselben mit der Haut in Verbindung setzten. Es ist dies nicht richtig. Der Quadratus inf. schickt gar nicht wenige seiner Faserziigee durch den Orbicularis hindurch rïckwärts zur Schleimhaut in der Niihe des Lippenrandes (Fig. 2). Vom Quadratus sup. wollte es mir nicht gelingen, in dieser Hinsicht ein völlig unzweideutiges Bild zu gewinnen. Ein gleich genauer zu schilderndes Fasersystem wirkt hier allzu störend. Dagegen verflechten sich beide Quadrati mit denjenigen Parthien des Orbicularis, die nach vorn zur Haut umbiegen. Es sind ibre medialen Faserbündel, die von diesem Schicksale betroffen werden.

Wir haben es bisher mit allbekannten Faserzügen zu thun gehabt und unsere Aufgabe bestand wesentlich nur darin, deren Anordnung und Verlaufsweise genauer, als es nach den bisher gebräuchlichen Methoden geschehen konnte, festzustellen. Sie erzeugen den hauptsächlichsten, doch keineswegs den einzigen Bestandtheil des Orbicularis. Ihnen zur Seite tritt noch ein eigenthümliches, reiches System von Muskelbiundeln, die mit völliger Umgehung des Skeletes nahe dem Lippenrande in gestrecktem, vorherrschend sagittalem Verlaufe durch den Orbicularis hindurch die Haut direct mit der Schleimhaut verknüpfen. Ohne Anwendung des Mikroskopes muissen sie als austretende Elemente 
des Orbicularis angesehen werden und die so vielfach, namentlich auch von Langer, aufgestellte Behauptung, dass letzterer in der ganzen Lippenbreite mit der Haut in Verbindung trete, ist hauptsächlich auf sie zurückzuführen.

In der Literatur finde ich ihrer an einer einzigen Stelle Erwälnung gethan. Klein ${ }^{1}$ ) theilt mit, dass man sich durch gut gefiihrte 'Schnitte überzeugen könne, wie ein Theil der Muskelfasern, welche in die Cutis aus dem Orbicularis übertreten, einem eigenen System angehören, dessen Elemente in den Zwischenräumen der ersten 5-7 ïbereinanderstehenden Reihen von Haarbälgen beginnen, sich im subcutanen Gewebe zu 4-5 Bündelchen ordnen und in sehr mässig gekrümmtem Bogen zwischen den Bündeln des Sphincter hindurchziehen, um schliesslich fächerförmig in die Schleimhaut selbst, selten in deren Uebergangstheil einzugehen. Kle in bezeichnet das System als Compressor labii, spricht sich übrigens ibber dessen genaueres Verhalten nur so weit aus, dass er es in der Medianlinie der Unterlippe stärker ausgebildet sein lässt als an den Seitentheilen, was für die Oberlippe, wo es überhaupt schwächer sei, in umgekehrtem Sinne gelte. An den Seitentheilen soll seine Richtung radiär zur Mundspalte sein.

Diese Angaben bedürfen in mehrfachen Punkten der Erweiterung und theilweise anch der Berichtigung. Sie reichen keineswegs hin, um uns ein vollständiges Bild dieses so lange übersehenen Lippenmuskels zu verschaffen. Namentlich lassen sie vollständig darüber im Unklaren, in welcher Ausdehnung austretende Orbicularisfasern durch sie vorgetäuscht werden. Darauf kommt es aber gerade an; denn obgleich der Darstellung durch das Scalpell unzugänglich, so spielt die ganze Fasergruppe doch sicher im Mechanismus der Lippen eine keineswegs unwichtige Rolle, indem sie dieselben der Dicke nach zusammenpresst. An und für sich ist daher anch gegen die von Klein gewählte Benennung eines Compressor labii nichts einzuwenden und nur der Wunsch, überhaupt mit allen, zum Theil völlig unzutreffenden, wenn" gleich allgemein gebräuchlichen, physiologischen Namen der Mundmuskeln aufzuräumen, veranlasst mich, eine andere und zwar diejenige eines geraden Lippenmuskels (M. Rectus labii) vorzuschlagen.

1) Stricker, Handbuch der Lehre von den Geweben des Menschen und der Thiere. Leipzig, 1871. 1. Bd. S. 359. 
Derselbe gehört dem Randtheile der beiden Lippen in deren ganzen Breite an und iberschreitet ihn seitlich nach der Wange hin nur um weniges. Im Gegensatze zu all seinen Nachbarn entbehren seine Faserbündel des geschlossenen Bestandes. Sie ordnen sich in kurzen Abständen $\mathrm{zu}$ fortlaufender, ziemlich dichter Reihe (Fig. 4). Sie durchsetzen dabei sämmtlich platt oder rundlich den Randtheil des Orbicularis, soweit derselbe von durchgehenden Fasern gebildet wird und täuschen gerade dadurch einen Austritt der letzteren nach der Haut hin vor. Zunächst der Medianebene ist ihr Verlauf ein schräger, nach dor Schleimhaut hin absteigend an der Oberlippe, in gleicher Richtung aufsteigend an der Unterlippe (Fig. 1-3). Ihr Hautende reicht rom ersten Haarbalge bis zum 10. oder 14. hin. In der Schleimhaut rícken sie von den ersten Traubendrïsen, zwischen welchen sie zum Theil durchtreten, bis zum höchsten Punkte der Lippen vor. Gegen den Mundwinkel zu wird ihr Verlauf mehr und mehr ein sagittaler, indem sie auf Seiten der Schleimbaut nicht mehr so nahe an die Mundspalte herantreten. Ám Mundwinkel selbst treffen sie auf die nach vorn biegenden Fasern des Zygomaticus und Buccinatorius und können bei oberfäichlicher Betrachtung leicht damit verwechselt werden. Hier namentlich laufen sie nicht selten mehr oder weniger schräg oder wohl auch gebogen von vorn nach hinten; die von Kle in behauptete radiäre Anordnung habe ich inclessen nie beobachtet. Ebensowenig kann ich ihm beistimmen, wenn er den Muskel der Oberlippe für im ganzen schwächer als den der Unterlippe erklärt. Ich habe nicht selten den entgegengesetzten Eindruck gehabt, will indessen auf eine allfällige Grössenverschiedenheit überhaupt kein besonderes Gewicht legen. Seine Stärke nimmt an beiden Lippen von der Mitte nach den Enden hin, doch nur sehr langsam, ab. In gleichem Maasse sammeln sich seine anfangs zerstreuten Bündel zu einer mehr einheitlichen Masse. Auch jenseits des Mundwinkels bleiben beide Muskeln streng von einander geschieden.

Die Fasern des Rectus labii verflechten sich auf der Hautseite innig mit den Enden der beiden Quadrati (Fig. 2 u. 3). Wo Theile derselben, wie an der Unterlippe, statt zur Hant, durch den Orbicularis hindurch zur Schleimhaut gehen, geschieht dies stets zunächst den dem Lippenrande fernsten Bündeln des Rectus. 
Gerade daraus erwächst die Schwierigkeit, die beiden Fasersysteme in allen Fällen streng auseinanderzuhalten.

Sammeln wir schliesslich die einzelnen Striche unserer Darstellung zu einem einheitlichen Bilde, so fâllt dasselbe freilich von all den bisher qer Mundmuskulatur zugeschriebenen verschieden genug aus.

Vor allem muss nochmals hervorgehoben werden, dass der Orbicularis, wenngleich in einer ganz anderen Richtung, als man bisher so vielfach angenommen hat, im Rectus labii einen wirklichen Eigenmuskel besitzt, der seine Grenzen nach keiner Seite hin überschreitet. Seine Hauptmassen und seine morphologisch wie physiologisch grundlegenden Elemente bezieht er allerdingrs von aussen her. Ihrem Verlauf nach scheiden sich diese wieder in solche mit und solche ohne Kreuzung. Bei letztern ist des weitern darauf zu achten, ob die Kreuzung nur in vertikaler Richtung am Mundwinkel, oder auch der Quere nach in der Mitte der Lippen stattfindet. Mit Berücksichtigung dieser Punkte lässt sich folgende Tabelle aufstellen:

1) Muskeln mit Kreuzung der Fasern.

a. Einfache Kreuzung an Mundwinkel: Buccinatorius.

b. Doppelte Kreuzung am Mundwinkel und in der Lippenmitte: Triangularis sup. und inf.

2) Muskeln ohne Kreuzung der Fasern. Quadratus sup. und inf. - Zygomaticus. - Risorius.

Characteristische Unterschiede zeigt auch die Endigungsweise. Die einfach gekreuzten Fasern kümmern sich weder um die Haut noch um die Schleimhaut, die doppelt gekreuzten wenden sich einseitig der Haut zu. Die ungekreuzten allein lassen Haut und Schleimhaut gleichzeitig, immerhin mit Bevorzugung der ersteren, ihre Fürsorge angedeihen. Sie verlegen dabei das Feld ihrer Thätigkeit vorzugsweise anf das Ende der Lippen und iiberlassen deren Mitte ihren doppelt gekreuzten Genossen.

Von besonderer Wichtigkeit ist, dass die Triangulares den freien Lippenrand ausschliesslich dem Besitze des Buccinatorius und der ungekreuzten Fasern überlassen. Sie bilden gleichsam einen äusseren Rahmen, der nach der Mundspalte zu ron den ibrigen Muskeln überragt wird. Diesen Randtheil durchspicken dann noch in sagittaler Richtung die zahlreichen Bündel des Rectus. Das 
nachfolgende Schema ist geeignet, diese Sachlage in möglichst durchsichtiger Weise zu veranschanlichen.

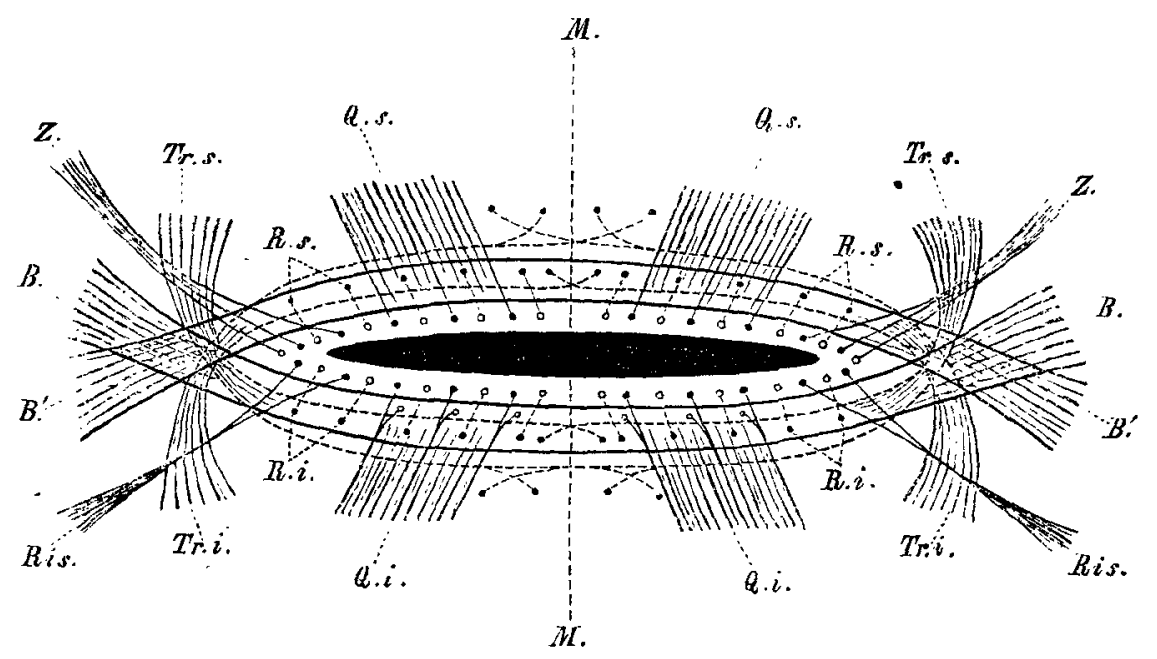

Schematische Darstellung der Mundmuskulatur.

MXI Medianebene; BB' Buccinatorius; Z Zygromaticus; Ris Risorius; Tr s Triangularis sup.; Tr i Triangularis inf.; R s Rectus labii sup.; R i Rectus labii inf.; Q s Quadratus sup.; $Q$ i Quadratus inf. - Die dunkeln Kreise entsprechen den Ansätzen an der Haut, die hellen denjenigen an der Schleimhant. - Anf die spezielle Topographie wurde in Interesse der Uebersichtlichkeit keine Rücksicht genommen.

Aus der geschilderten Anordnung der Muskeln ergeben sich die physiologischen Schlussfolgerungen von selbst. Einige der wichtigsten Punkte verdienen immerhin besonders namhaft gemacht zu werden. Die Mundöffnung besitzt in den Triangulares ein System ron circulären, in den Quadrati, dem Zygomaticus und Risorius ein solches von radiären Fasern. Beide sind reine Antagonisten, jene im Sinne von Verengern, diese in solchem von Erweiterern. Die Viertheilung des circulären Fasersystems mit streckenweiser Verschränkung der benachbarten Gruppen gestattet ebensowohl ein einheitliches Zusammenwirken als auch gesonderte Thätigkeit der einzelnea Bezirke. Durch verschiedenartige Combination mit den radiären Muskeln wird die Mannigfaltigkeit der zu erzielenden Nutzeffecte natïrlich um ein bedeutendes gesteigert. Der Buccinatorius behauptet eine Mittelstellung. Er verengert die 
Mundöffnung in senkrechter und erweitert sie in querer Richtung. Gleichzeitig rollt er in Folge seiner starken Biegung am Mundwinkel den Lippenrand nach hinten. Er wird hierin durch den Zygomaticus unterstützt. Er gestaltet sich dadurch zu einem Antagonisten für die übrigen radiären Muskeln, welche die Lippen nach aussen umkrempen. Es ist bedeutungsvoll, dass der freie Lippenrand sich im ausschliesslichen Besitze dieser beiden Fasergruppen befindet und die eigentlichen Schnürer erst in grösserer Entfernung auftreten. Der Rectus labii besitzt wohl kaum eine selbständige Thätigkeit. Dagegen ist er jedenfalls im Stande, die Prallheit und Festigkeit des Lippenkörpers durch seine Beihülfe zu fördern.

Nach dem Gesagten bedarf.es wohl kaum des besondern Hinweises darauf, wie völlig verkehrt die Bezeichungen der beiden Triangulares als Heber und Senker des Mundwinkels sind, wie sehr es geboten ist, sie ein für allemal zu beseitigen. Man hat für den Buccinatorius den Ausdruck des Buccolabialis vorgeschlagen. Nach dem Verlaufe seiner Fasern gewiss mit vollem Rechte. Nichtsdestoweniger halte ich es für besser, bei dem alten Ausdrucke des Buccinatorius zu verbleiben. Denn legt man einmal Gewicht darauf, dem Verhalten der Muskelfasern in ihrer ganzen Ausdehnung Rechnung zu tragen, so ist doch gewiss kein Grund vorhanden, solches nur dem Buccinatorius und nicht auch den Triangulares gegenuiber zu thun. Man müsste dann auch diese anders benennen und ihnen beispielsweise nach dem Vorschlage ron Henke die Bezeichnung der Circumflexi beilegen. Ich bezweifle, dass damit viel gewonnen wäre, und bin der Meinung, dass dem praktischen Bedürfnisse einer verständlichen Darstellung durch die Beibehaltung eines Orbicularis und von aussen an denselben herantretender Muskeln ein weit besseres Genüge geleistet wird. Die Triangulares und der Buccinatorius werden nach wie vor fortfahren, vor ihrem Eintritte in den muskulösen Randwall der Mundöffnung so gut wie die Quadrati und Genossen den Eindruck selbständiger Muskelindividuen zu machen. Ihn zu verflachen dürfte mehr Nachtheil als Vortheil bringen.

Ich will nicht schliessen, ohne nochmals auf das grosse Interesse hinzuweisen, welches die vergleichend anatomische Untersuchung der Muskulatur der Mundspalte bieten muisste. Was bisher darin geleistet worden, genügt kaum den bescheidensten An- 
sprichen. Eine wissenschaftliche Physiognomik auf anatomischer Grundlage muss aber so lange frommer Wunsch bleiben, als das Mikroskop nicht Licht in das noch vorhandene Dunkel gebracht und die bisher benutzten verschwommenen Umrisse durch klar und scharf gezogene Linien ersetzt hat.

\section{Erklärung der Abbildungen anf Tafel XXXIV.}

Fig. 1. Medianschnitt der Unterlippe.

Fig. 2. Senkrechter Dickenschnitt der Unterlippe seitlich von der Medianebene. Fig. 3. Senkrechter Dickenschnitt der Oberlippe seitlich von der Medianebene. Fig. 4. Horizontaler Dickenschnitt durch die Mitte der Unterlippe in geringer, Fig. 5. in grösserer Entfernung vom freien Lippenrande.

Fig. 6. Horizontaler Dickenschnitt durch den Mrundwinkel.

c Cutis mit Haarbälgen. - m Mucosa, in Fig. 1-3 mit Schleimdrüsen (gl). $-B$ Buccinatorius. - Tr Triangularis. $-Q$ Quadratus. R Rectus. - Z Zygomaticus. - Ris Risorius.

Sämmtliche Figuren sind vermittelst des Scioptikons mit vorgesetztem Prisma in ihren Grundzügen genau nach den Präparaten entworfen und dann an der Hand des Mikroskopes in den Einzelheiten ausgearbeitet worden. 